\title{
Influence of the 6-month physical activity programs on renal function in obese boys
}

\author{
Irina Lousa ${ }^{1}$, Henrique Nascimento ${ }^{2}$, Susana Rocha ${ }^{2}$, Cristina Catarino ${ }^{2}$, Flávio Reis ${ }^{3}$, Carla Rêgo ${ }^{4}$, Alice Santos-Silva ${ }^{2}$,
} André Seabra $^{5}$, Sandra Ribeiro ${ }^{2}$ and Luís Belo ${ }^{2}$

BACKGROUND: We intended to evaluate the effects of physical activity (PA) programs on renal function in obese boys.

METHODS: Thirty-nine boys participated in one of the following three groups: soccer ( $S G, n=13)$, traditional PA (AG, $n=13)$, and sedentary control $(C G, n=13)$. SG and $A G$ were involved in 6-month PA programs, involving three sessions/week for 60-90 min. Anthropometric measurements, body composition, creatinine and cystatin C plasmatic levels, and estimated glomerular filtration rate (eGFR) were evaluated. RESULTS: At baseline $(n=39)$, age and lean mass index (LMI) were positively correlated with creatinine levels. After 6 months, both intervention groups decreased the BMI $z$-score and waist circumference, while the CG increased the body fat percentage (BFP). LMI increased in all the groups. SG presented a small increment in plasma creatinine and a decrease in the eGFR values, using the Schwartz formula. Concerning the cystatin C levels and eGFR values using Filler (cystatin C-based) or Combined Zappitelli (creatinine/cystatin C-based) formulas, no significant changes were observed in any group.

CONCLUSION: The combined Zappitelli formula showed no significant impact of PA on eGFR in obese boys. Although plasma creatinine is significantly influenced by lean body mass, cystatin $C$ is likely to be a more accurate marker of renal function in this population.

O besity is a chronic disease that is growing worldwide, reaching a particularly alarming rate of increase in childhood (1). Childhood obesity is associated with several comorbidities and cardiometabolic risk factors, namely dyslipidemia, insulin resistance, and low-grade inflammation (2). High values of acute-phase proteins (e.g., C-reactive protein), of insulin-resistance index, and of triglycerides can be observed in obese children and adolescents (2). Obesity is a risk factor for the development of kidney disease (3), and recent data also suggest that some degree of renal impairment may be observed in overweight and obese children (4). A work performed in young prepubertal children demonstrated significantly lower estimated glomerular filtration rate (eGFR) in those who were overweight/obese, compared with nonoverweight controls, even though all the participants presented renal function markers and eGFR within the normal range (4).

Physical activity (PA) is an important tool for obesity prevention and treatment in childhood, as regular PA improves body composition, cardiorespiratory fitness, and reduces cardiovascular risk factors in obese children and adolescents $(5,6)$. Improvements in the adipokine profile, decreasing inflammatory substances, and increasing adiponectin (which has several protective properties) have been reported in interventional studies involving physical exercise. Furthermore, these modifications occur concomitantly with beneficial cardiometabolic changes, such as increment in high-density lipoprotein levels, and reductions in blood pressure and insulin resistance index $(5,6)$. Renal function also seems to improve after weight loss in obese patients (7). However, little data exist regarding renal changes with PA in obese patients at pediatric ages (8).

This study aimed to investigate and compare the effect of 6month soccer and traditional PA programs on renal function in obese boys.

\section{METHODS}

\section{Subjects}

This study was part of a larger project that aimed to study the impact of soccer and traditional PA programs on the changes in body composition and cardiometabolic risk factors in obese boys (6). We analyzed a subsample of the patients enrolled for that study, including 39 boys distributed in the following three groups: soccer (SG, $n=13$ ), traditional physical activity (AG, $n=13$ ), and sedentary control (CG, $n=13$ ).

The detailed description of patients is described elsewhere (6). Briefly, boys were recruited from an outpatient clinic of pediatric obesity in Oporto. Inclusion criteria were: age $8-12$ years, obese (body mass index $(\mathrm{BMI})>2$, standard deviations above age- and sex-specific WHO reference medians (9)), and no medication or

\footnotetext{
'Department of Biological Sciences, Laboratory of Biochemistry, Faculty of Pharmacy, University of Porto, Porto, Portugal; ${ }^{2}$ UCIBIOVREQUIMTE, Department of Biological Sciences, Laboratory of Biochemistry, Faculty of Pharmacy, University of Porto, Porto, Portugal; ${ }^{3}$ Laboratory of Pharmacology and Experimental Therapeutics, Coimbra Institute for Clinical and Biomedical Research (iCBR), Faculty of Medicine and CIBB Consortium, University of Coimbra, Coimbra, Portugal; ${ }^{4}$ Children and Adolescent Centre, CUF Hospital, Center for Health Technology and Services Research (CINTESIS), Faculty of Medicine, University of Porto, Porto, Portugal; ${ }^{5}$ Research Centre in Physical Activity, Health and Leisure (CIAFEL), Faculty of Sport, University of Porto, Porto, Portugal. Correspondence: Luís Belo (luisbelo@ff.up.pt)

Received 5 September 2017; accepted 11 January 2018; advance online publication 11 April 2018. doi:10.1038/pr.2018.15
} 
diagnosed medical conditions that could affect the study results or limit the PA. Boys who could not participate in the interventions or who were not interested, acted as a control group (CG).

The study was ethically approved by the research committee of the Faculty of Sport of the University of Porto and by the hospital authorities. It was conducted in compliance with the Declaration of Helsinki. All boys participated in the study after the informed and written consent of their parents or legal representatives.

The 6-month interventions implemented with SG and AG included three sessions per week of 60-90 min duration. The soccer program consisted of warm-up session (10-20 min), technical drills and small-sided games (40-60 min), and cool-down session (10 min). It was conducted at a local soccer club on Tuesdays, Thursdays (1830-2000), and Saturdays (1000-1130). The traditional activity program was multidimensional with a variety of exercise routines, consistent with international recommendations. Each session included warm-up (10-20 min), generalized activities aimed at improving aerobic endurance, coordination, balance, flexibility and strength, e.g., walking, running, gymnastics, and others (40$60 \mathrm{~min})$, and cool-down $(10 \mathrm{~min})$. The intervention was carried out at a school on Mondays, Wednesdays, and Fridays (1830-2000). Training intensities for the SG and AG interventions were designed to maintain the heart rate (HR) at $\sim 70-80 \%$ of the maximum (HRmax), confirmed by monitoring (Polar Team 2 Pro System, Polar Electro, Kempele, Finland). All intervention sessions were conducted by two physical education teachers, under the guidance of the principal investigator. Sessions were initiated on 9 September 2013 and concluded on 9 March 2014.

\section{Anthropometry}

Body mass was measured with a digital scale (Tanita, London, UK, BC-418MA); height was measured with a fixed stadiometer (Holtain, Crymych, UK); and the waist circumference (WC) was measured with a metallic tape (Holtain). All were measured following the standard procedures. BMI was calculated by dividing body weight in kilograms by the square of height in meters. The $z$-score values for BMI were obtained using the WHO AnthroPlus software (WHO anthro).

\section{Body Composition}

Body composition was measured by dual-energy X-ray absorptiometry (DXA) (Hologic QDR 4500A, Hologic, Waltham, MA). The unit was calibrated according to the manufacturer instructions; a trained technician did all the exams. Body fat percentage (BFP) and lean mass were measured using standard protocols. Lean mass index (LMI) was calculated by dividing the lean mass in kilograms by the square of height in meters. The same investigator analyzed all the body scans.

\section{Renal Function Markers}

After an overnight fast, blood samples were collected by venipuncture in EDTA, containing tubes and processed within $2 \mathrm{~h}$ of collection. Samples were collected at baseline and after 6 months between 08:00 and 10:00 hours at the Faculty of Sport of the University of Porto.

Aliquots of plasma were made and stored at $-80{ }^{\circ} \mathrm{C}$, until assayed. Baseline and follow-up samples were analyzed simultaneously. Plasma creatinine was evaluated using the standardized kinetic method of alkaline picrate-kinetic in an automatic analyzer Beckman Coulter AU5800 (Beckman Coulter, Brea, CA, USA), with linearity between 0.06 and $25.0 \mathrm{mg} / \mathrm{dl}$. Plasma cystatin C levels were determined using a commercially available enzyme-linked immunoassay, Quantikine Human Cystatine C Immunoassay (R\&D Systems, USA).

To estimate the eGFR $\left(\mathrm{ml} / \mathrm{min} / 1.73 \mathrm{~m}^{2}\right)$, the following formulas were used: Revised Schwartz formula (Schwartz-R) $-k \times$ (height

Table 1. General characteristics, renal function markers, and estimated glomerular filtration rates, at baseline and after 6 months, in the three obese groups

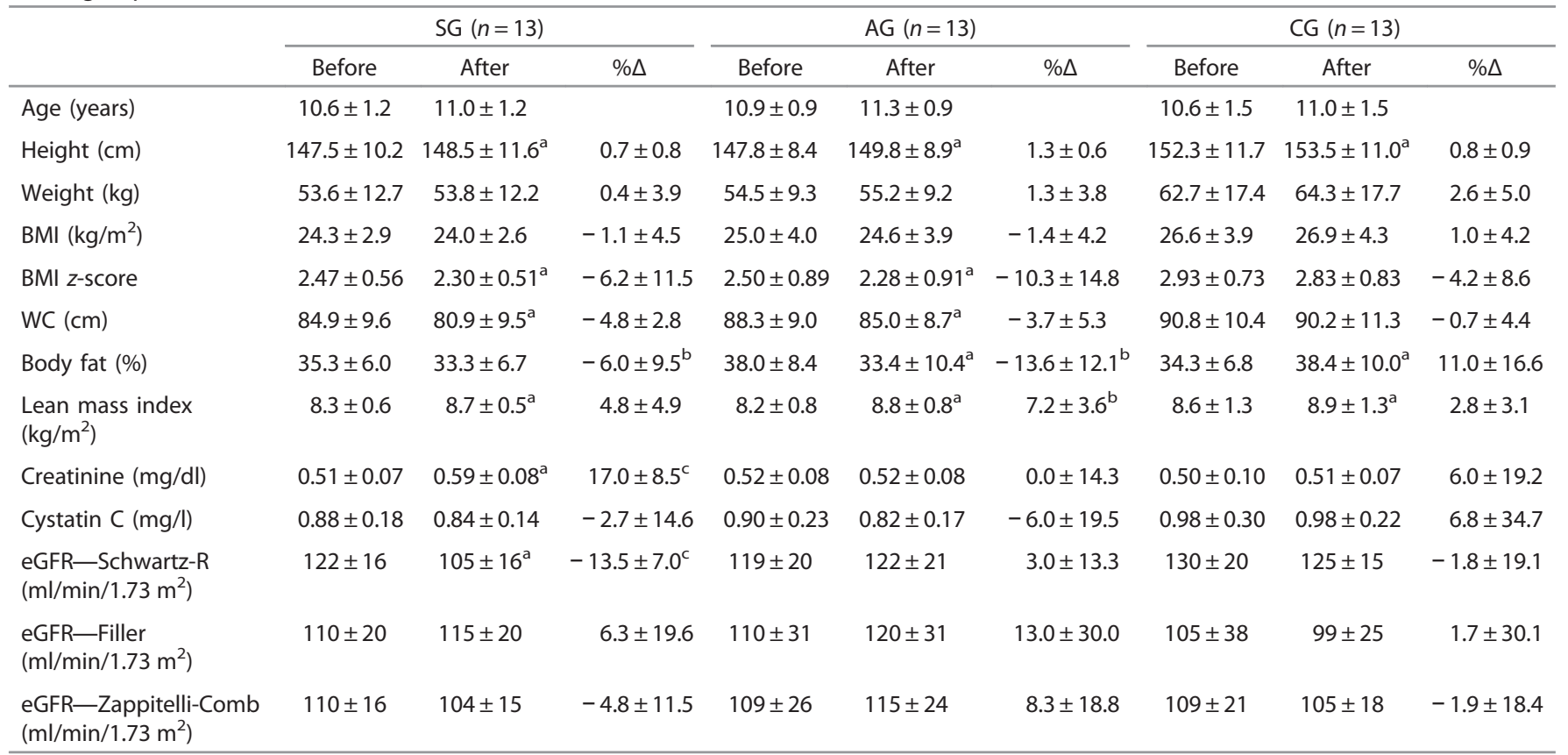

Results are presented as mean \pm SD.

AG, traditional physical activity group; BMI, body mass index; CG, control group; WC, waist circumference; eGFR, estimated glomerular filtration rate; Filler, Filler formula; Schwartz-R, Revised Schwartz formula; SG, soccer group; Zappitelli-Comb, Combined Zappitelli formula.

a ${ }^{2}$ ignificant baseline to post difference within the group $(P<0.05)$.

${ }^{b} \%$ Change significantly different from CG $(P<0.05)$.

$c_{\%}$ Change significantly different from $A G(P<0.05)$. 
$(\mathrm{cm}) /$ serum creatinine $(\mathrm{mg} / \mathrm{dl}))$, using a $k$ constant of $0.413(10)$; Filler formula $-\log ($ GFR $)=1.962+(1.123 \times \log (1 /$ cystatin $C(\mathrm{mg} / \mathrm{l}))$ (11); Combined Zappitelli formula (Zappitelli-Comb) $\left(507.76 \times \mathrm{e}^{0.003 \times \text { height }(\mathrm{cm})}\right) /\left(\right.$ cystatin $\mathrm{C}(\mathrm{mg} / \mathrm{l})^{0.635} \times$ serum creatinine $\left.(\mu \mathrm{mol} / \mathrm{l})^{0.547}\right)(12)$.

\section{Statistical Analysis}

Statistical analysis was performed, using the IBM Statistical Package for Social Sciences (SPSS, version 24.0, Chicago, IL) for Windows. Kolmogorov-Smirnov analysis was used to test whether the results were normally distributed. Differences between the groups at baseline were tested using chi-squared test and Fisher's exact test for categorical variables; for continuous variables, multiple comparisons between groups were performed by one-way ANOVA supplemented with Tukey's HSD post hoc test. The effect of the PA intervention programs, relative to the CG, was evaluated with a two-factorial repeated measures ANOVA, using the Group (SG, AG, $\mathrm{CG})$ as "Between-Subjects Factor" and Time (pre, post) as "WithinSubjects Variable". For each variable, the percentage change $(\% \Delta)$ was calculated between values at baseline and 6 months. The between-group differences in pre-post delta values were evaluated by one-way ANOVA. Pearson correlation coefficient was calculated to evaluate the relationships between the sets of data. A $P$ value lower than 0.05 was considered as statistically significant.

\section{RESULTS}

An average of 72 sessions was conducted in SG and AG. Average number of sessions attended (adherence) was $>85 \%$ for both groups. No significant differences between SG and AG were found for average training duration $(84.4 \pm 6.5$ vs. $80.1 \pm 8.3 \mathrm{~min} /$ session, respectively) and maximum heart rate ( $\approx 78 \%$ vs $75 \%$, respectively).

Characteristics of the participants and renal function markers at baseline and after 6 months are summarized in Table 1. The results presented a Gaussian distribution, and therefore are presented as mean \pm standard deviation. At baseline, no significant differences were observed between the three groups, for clinical or laboratory data.

Boys in all the groups increased significantly in height $\left(F_{(1,36)}=56.44, \quad P<0.001, \eta_{\mathrm{p}}{ }^{2}=0.61 \%\right)$. Despite significant Group $\times$ Time interactions, increments were also observed in all the groups for LMI (SG: $F_{(1,36)}=19.17, P<0.001$, $\eta_{\mathrm{p}}{ }^{2}=0.35 \% ;$ AG: $F_{(1,36)}=43.5, P<0.001, \eta_{\mathrm{p}}{ }^{2}=0.55 \%$; CG: $\left.F_{(1,36)}=7.61, P=0.009, \eta_{\mathrm{p}}{ }^{2}=0.18 \%\right)$.

After 6 months, both intervention groups presented a significant decrease in BMI $z$-score (SG: $F_{(1,36)}=6.37$, $P=0.016, \quad \eta_{\mathrm{p}}^{2}=0.15 \% ; \quad$ AG: $\quad F_{(1,36)}=11.17, \quad P=0.002$, $\left.\eta_{\mathrm{p}}{ }^{2}=0.24 \%\right)$ and waist circumference (SG: $F_{(1,36)}=15.83$, $P<0.001, \quad \eta_{\mathrm{p}}^{2}=0.31 \% ; \quad$ AG: $\quad F_{(1,36)}=11.12, \quad P=0.002$, $\left.\eta_{\mathrm{p}}{ }^{2}=0.24 \%\right)$. A significant Group $\times$ Time interaction was present for BFP, with $\mathrm{AG}$ decreasing the BFP during the course of intervention $\left(F_{(1,36)}=12.80, P=0.001, \eta_{\mathrm{p}}{ }^{2}=0.26 \%\right)$,
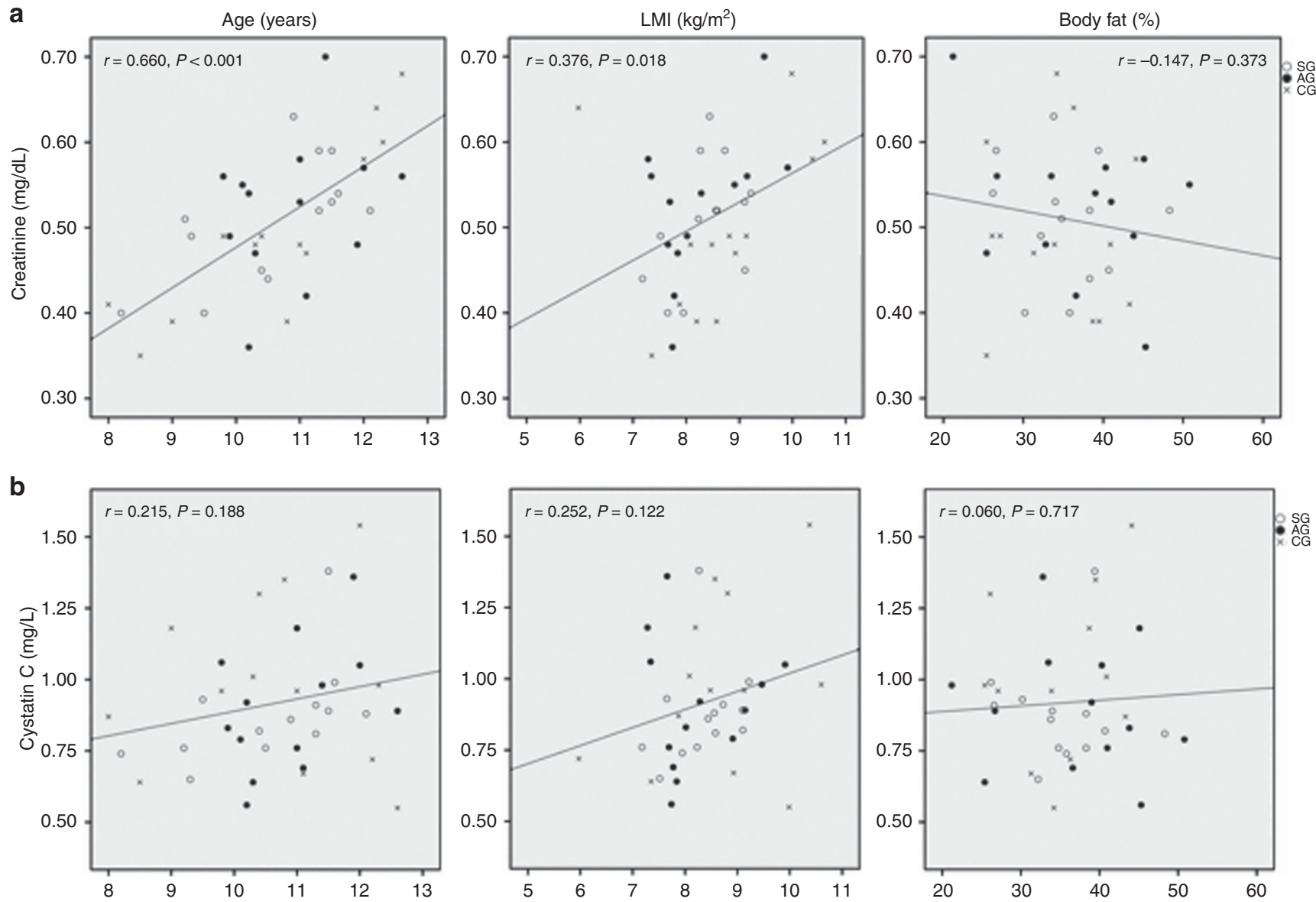

Figure 1. Correlations between plasma levels of creatinine (a) and cystatin C (b) with age, lean mass index (LMI), and body fat percentage of obese participants at baseline $(n=39)$. $r$, Pearson correlation coefficient; AG, traditional physical activity group; CG, control group; SG, soccer group. 


\section{Articles $\mid$ Lousa et al.}

whereas CG increased the BFP (SG: $F_{(1,36)}=11.92, P=0.001$, $\left.\eta_{\mathrm{p}}{ }^{2}=0.25 \%\right)$; SG also reduced the $\mathrm{BFP}$, reaching borderline statistical significance $\left(F_{(1,36)}=2.98, P=0.093, \eta_{\mathrm{p}}{ }^{2}=0.08 \%\right)$. Pre-post delta values were different between intervention groups and CG for BFP (SG vs. CG: $P=0.006$; AG vs. CG: $P<0.001)$, and between AG and CG for LMI $(P=0.019)$.

Renal function markers were similar across the three groups at baseline (Table 1). A significant increment in creatinine levels $\left(F_{(1,36)}=18.35, P<0.001, \eta_{\mathrm{p}}{ }^{2}=0.34 \%\right)$ and a decrease in eGFR values using the Schwartz formula $\left(F_{(1,36)}=10.99\right.$, $\left.P=0.002, \eta_{\mathrm{p}}{ }^{2}=0.23 \%\right)$, were observed in SG. Concerning cystatin $\mathrm{C}$ levels and eGFR values using Filler or Combined Zappitelli formulas, no interactions $\left(F_{(2,36)}=0.49, P=0.619\right.$; $F_{(2,36)}=0.99, P=0.380 ; F_{(2,36)}=1.91, P=0.162$, respectively) or main effects for time $\left(F_{(1,36)}=1.23, P=0.275 ; F_{(1,36)}=0.41\right.$, $P=0.526 ; F_{(1,36)}=0.16, P=0.692$, respectively) were noted. Pre-post delta values were only different between SG and AG for creatinine $(P=0.015)$ and for eGFR, using the Schwartz formula $(P=0.013)$.

At baseline $(n=39)$, age and LMI values were positively and significantly correlated with creatinine levels (Figure 1). Changes in creatinine levels (pre-post delta values) showed no statistically significant correlations with changes in LMI or BFP.

\section{DISCUSSION}

As far as we know, this is the first study assessing the influence of PA on renal function in obese children and adolescents.

At baseline, the groups were matched for clinical and laboratory data allowing comparisons between them. Gender was not a confounding factor, as we only studied males. Actually, the evaluation of the potential use of soccer, which is a more attractive sport for males, as a therapy for obesity led us to limit the intervention to males (6).

The results of the present study confirm that PA (either soccer or traditional activity) is an important tool for the treatment of obesity. Over the 6-month intervention, SG and AG improved the anthropometric measurements and body composition. It is important to emphasize that soccer is a popular sport (13), relatively inexpensive and accessible to all, and has the potential to be explored as an attractive tool for the prevention and the treatment of obesity.

In clinical practice, circulating creatinine is the most commonly used biomarker of renal function. Plasma creatinine is known to be relatively independent of protein ingestion, water intake, and rate of urine production, but it can be affected by factors such as ethnicity, gender, age, and lean mass. Our data confirm the influence of age and lean mass on plasma creatinine levels. On the other hand, we found no significant association between the degree of obesity and markers of renal function. Previous studies reported contradictory results regarding this matter, with some authors describing a positive association between the magnitude of obesity and the renal eGFR (14), whereas others reported lower eGFR (4) or absence of any differences (15) in overweight/obese children, compared with nonoverweight controls.

The SG group presented a significant increment in plasma creatinine and a reduction in eGFR, when using a creatininebased prediction formula. The increment in circulating creatinine in this group was small, but consistent within individuals. Curiously, some individuals in AG group also presented increments in plasma creatinine, but the results were not consistent within individuals. It is likely that the type of PA may explain the increase in creatinine in SG (16-18).

The increase in plasma creatinine (SG group) was accompanied by increments in LMI, but these changes were not significantly correlated. Thus, the major contributing factor, explaining the changes in creatinine, seems to be other than LMI. In line with this, both AG and CG groups presented an increment in LMI (particularly the AG group), without significant changes in creatinine concentration.

Our results concerning creatinine are in agreement with those reported by Colombini et al. (18), who observed a postmatch reduction in eGFR in male soccer players. However, a decrease in renal function is unlikely to explain the observed increment in plasma creatinine in SG. Indeed, no significant differences were observed for plasma cystatin C or eGFR, when using a creatinine and cystatin C-based prediction formula. It is even worthy to emphasize the trend to increase the eGFR calculated with the Combined Zappitelli formula in the AG compared to the SG and CG. The trend to decrease cystatin $\mathrm{C}$ levels with PA may partially explain this result, but larger studies (in order to increase the power of the analysis) are needed to clarify this point. Also, further studies should present measurements of GFR (e.g., iohexol clearance) at baseline and at endpoint of the study, in addition to theeGFR.

We used Combined Zappitelli formula instead of other combined formulas, as previous results obtained with a Portuguese sample of overweight and obese children, demonstrated that this formula was the one that presented the closest performance to creatinine clearance (4). Also, when compared with inulin clearance, the Zappitelli formula presented (amongst other formulas) the highest 30\% accuracy at measured GFR $\geq 90 \mathrm{ml} / \mathrm{min}$ per $1.73 \mathrm{~m}^{2}$ (19). Although, our cystatin $C$ levels were higher, and the eGFR values were lower than those previously reported (4). This is probably due to the different analytical methods used to measure cystatin C, as we used the ELISA technique instead of an automatized particle-enhanced immunonephelometric assay. Moreover, we are aware of the possible influence of our method to evaluate creatinine, on absolute values using the Schwartz formula; however, as we performed a follow-up analysis, this is minimized.

The present study had some limitations. Participants were not randomly assigned to the respective groups. Also, due to logistic constraints, the number of patients in each group was small, reducing statistical power analysis. Finally, we did not include nonoverweight controls, limiting the analysis of the association between obesity and renal function markers. 
In summary, the combined analysis of plasma levels of creatinine and cystatin $\mathrm{C}$ showed that 6-month PA programs had no significant impact on the renal function in obese boys. Caution should be made when using sole-based creatinine equations, as creatinine levels are influenced by internal factors, such as age and LMI. Soccer increased slightly the creatinine levels, though it was not correlated with changes in the body composition. Cystatin C may work as a more independent and accurate marker of renal function in sports, in the pediatric population. The study of longer PA interventions on renal function, involving larger sample sizes and a wider age-range obese pediatric patients is worthy.

\section{STATEMENT OF FINANCIAL SUPPORT}

This work received financial support from the Union European Football Association (UEFA), the Portuguese Football Federation (FPF), the European Union (FEDER funds POCI/01/0145/FEDER/007728), National Funds (FCT/MEC, Fundação para a Ciência e Tecnologia and Ministério da Educação e Ciência) under the Partnership Agreement PT2020 UID/MULTI/ 04378/2013, and Norte Portugal Regional Coordination and Development Commission (CCDR-N)/NORTE2020/Portugal 2020 (Norte-01-0145-FEDER-000024). We also thank the following support: UID/NEU/04539/2013, FCOMP-01-0124-FEDER-028417, and POCI-01-0145-FEDER-007440, as well as CENTRO-01-0145-FEDER-000012.

Disclosure: The authors declare no conflict of interest.

\section{REFERENCES}

1. GBD 2015 Obesity Collaborators, Afshin A, Forouzanfar MH, et al. Health effects of overweight and obesity in 195 countries over 25 years. N Engl J Med 2017;377:13-27.

2. Nascimento H, Costa E, Rocha-Pereira P, et al. Cardiovascular risk factors in Portuguese obese children and adolescents: impact of small reductions in body mass index imposed by lifestyle modifications. Open Biochem J 2012;6:43-50

3. Garofalo C, Borrelli S, Minutolo R, Chiodini P, De Nicola L, Conte G. A systematic review and meta-analysis suggests obesity predicts onset of chronic kidney disease in the general population. Kidney Int 2017;91:1224-35.

4. Correia-Costa L, Afonso AC, Schaefer F, et al. Decreased renal function in overweight and obese prepubertal children. Pediatr Res 2015;78:436-44.
5. Nascimento H, Alves AI, Medeiros AF, et al. Impact of a School-Based Intervention Protocol - ACORDA Project - on adipokines in an overweight and obese pediatric population. Pediatr Exerc Sci 2016;28:407-16.

6. Seabra A, Katzmarzyk P, Carvalho MJ, et al. Effects of 6-month soccer and traditional physical activity programmes on body composition, cardiometabolic risk factors, inflammatory, oxidative stress markers and cardiorespiratory fitness in obese boys. J Sports Sci 2016;34:1822-9.

7. Bolignano D, Zoccali C. Effects of weight loss on renal function in obese CKD patients: a systematic review. Nephrol Dial Transplant 2013;28 (Suppl 4): iv82-98.

8. Anke Doyon FS. The prodromal phase of obesity-related chronic kidney disease: early alterations in cardiovascular and renal function in obese children and adolescents. Nephrol Dial Transplant 2013;28:50-7.

9. de Onis M, Onyango AW, Borghi E, Siyam A, Nishida C, Siekmann J. Development of a WHO growth reference for school-aged children and adolescents. Bull World Health Organ 2007;85:660-7.

10. Schwartz GJ, Munoz A, Schneider MF, et al. New equations to estimate GFR in children with CKD. J Am Soc Nephrol 2009;20:629-37.

11. Filler G, Lepage N. Should the Schwartz formula for estimation of GFR be replaced by cystatin C formula? Pediatr Nephrol 2003;18:981-5.

12. Zappitelli M, Parvex P, Joseph L, et al. Derivation and validation of cystatin C-based prediction equations for GFR in children. Am J Kidney Dis 2006;48:221-30.

13. Dvorak J, Junge A, Graf-Baumann T, Peterson L. Football is the most popular sport worldwide. Am J Sports Med 2004;32:3S-4S.

14. Koulouridis E, Georgalidis K, Kostimpa I, Koulouridis I, Krokida A, Houliara D. Metabolic syndrome risk factors and estimated glomerular filtration rate among children and adolescents. Pediatr Nephrol 2010;25: 491-8.

15. Goknar N, Oktem F, Ozgen IT, et al. Determination of early urinary renal injury markers in obese children. Pediatr Nephrol 2015;30:139-44.

16. Randers MB, Nybo L, Petersen J, et al. Activity profile and physiological response to football training for untrained males and females, elderly and youngsters: influence of the number of players. Scand J Med Sci Sports 2010;20 (Suppl 1): 14-23.

17. Pohl AP, O'Halloran MW, Pannall PR. Biochemical and physiological changes in football players. Med J Aust 1981;1:467-70.

18. Colombini A, Machado M, Lombardi G, Lanteri P, Banfi G. Modifications of biochemical parameters related to protein metabolism and renal function in male soccer players after a match. J Sports Med Phys Fitness 2014;54:658-64.

19. de Souza V, Cochat P, Rabilloud M, et al. Accuracy of different equations in estimating GFR in pediatric kidney transplant recipients. Clin J Am Soc Nephrol 2015;10:463-70. 